\title{
Enhanced visible-light-driven photocatalytic performance of mesoporous W-Ti-SBA-15 prepared through a facile hydrothermal route
}

\author{
Fei Chang ${ }^{\mathrm{a}, *}$, Jie Wang ${ }^{\mathrm{a}}$, Jieru Luo ${ }^{\mathrm{a}}$, Junrong Sun ${ }^{\mathrm{a}}$, Baoqing Deng ${ }^{\mathrm{a}}$, Xuefeng Hu ${ }^{\mathrm{b}, *}$ \\ a School of Environment and Architecture, University of Shanghai for Science and Technology, Shanghai 200093, PR China \\ ${ }^{\mathrm{b}}$ Key Laboratory of Coastal Zone Environmental Processes and Ecological Remediation, Yantai Institute of Coastal Zone Research, Chinese Academy of \\ Sciences, Yantai, Shandong 264003, PR China
}

\section{H I G H L I G H T S}

- A series of tungsten-titaniumcocontained SBA15 composites were fabricated by a simple synthetic route.

- These samples showed enhanced catalytic performance in comparison to those comprising bare tungsten or titanium component.

- The enhancement of photocatalytic behavior under visible-light irradiation was discussed.

\section{A R T I C L E I N F O}

\section{Article history:}

Received 14 January 2016

Received in revised form 31 March 2016

Accepted 3 April 2016

Available online 4 April 2016

\section{Keywords:}

W-Ti-SBA15

Visible-light driven

Photocatalytic

Rhodamine B

2,4-Dichlorophenol

Mechanism
G R A P H I C A L A B S T R A C T

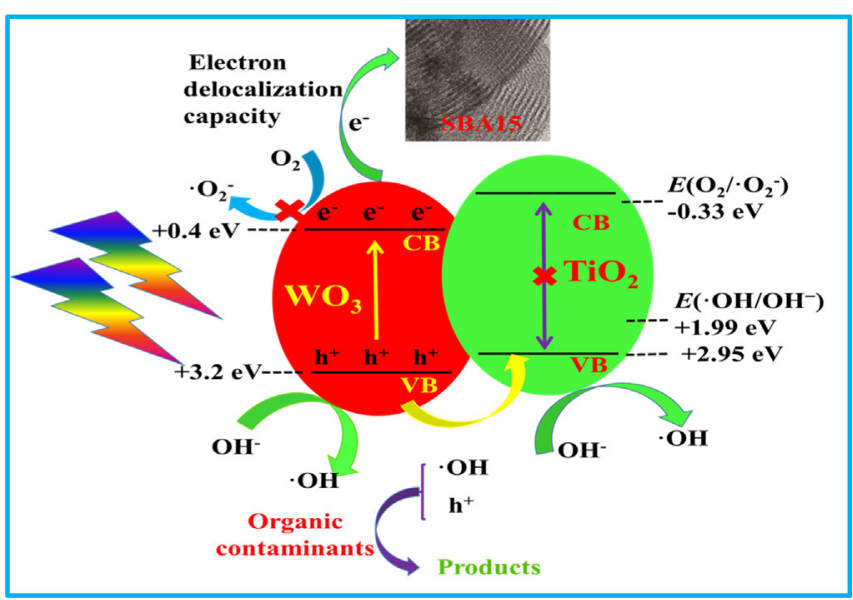

\begin{abstract}
A B S T R A C T
A facile and one-step hydrothermal procedure was adopted to fabricate a series of W-Ti-SBA15 composites (WTS). These as-prepared composites were systematically characterized by a collection of analytic and spectroscopic techniques. It was found that dual phases of titanium and tungsten species coexisted in mesoporous structures of $p 6 \mathrm{~mm}$ hexagonal symmetry. In addition, these composites were visible-light responsive and exhibited enhanced photocatalytic capability toward degradation of Rhodamine $\mathrm{B}(\mathrm{RhB})$ and 2,4-dichlorophenol (2,4-DCP) upon visible-light irradiation in comparison to samples containing single component of titanium or tungsten species. Specifically, the best candidate, sample 8\%WTS0.7, had the largest apparent reaction rate constant that was nearly 1.2, 2.1,2.2, and 5.7 times as high as those of $8 \% \mathrm{WS}, \mathrm{TS} 0.7, \mathrm{WO}_{3} / \mathrm{TiO}_{2}$, and $\mathrm{N}-\mathrm{TiO}_{2}$, respectively. The enhancement of photocatalytic performance was mainly attributed to the well-matched band structures of both components and strengthened visiblelight adsorption ability, originating from the combination of titania with highly acidic $\mathrm{WO}_{3}$ in $\mathrm{SBA} 15$ matrix with large specific surface areas. Active radical species were detected by trapping experiments and a possible photocatalytic mechanism was thus proposed.
\end{abstract}

(c) 2016 Elsevier B.V. All rights reserved.

\footnotetext{
* Corresponding authors.

E-mail addresses: feichang@usst.edu.cn (F. Chang), xfhu@yic.ac.cn (X. Hu).
} 


\section{Introduction}

In recent years, semiconductor-based photocatalysis has aroused tremendous attention due to its potential applications in fields of environmental remediation and renewable energy supply through a green redox process under moderate conditions[1-3]. Among numerous semiconductors researched, titania $\left(\mathrm{TiO}_{2}\right)$ is the most popular one in virtue of the favorable physicochemical properties and outstanding photocatalytic performance in ultraviolet region [4]. However, $\mathrm{TiO}_{2}$ is failed to trigger visible-light-driven photocatalysis because of the large band gap energy. In addition, high recombination of photoexcited hole-electron pairs and thus low quantum efficiency is another obstacle for pursuing practical uses of $\mathrm{TiO}_{2}$ [4]. To overcome abovementioned problems, several strategies such as structural modifications with metallic or nonmetallic elements, metal ions, dyes in terms of sensitization, and other semiconductors with narrow band gaps have been adopted to advance $\mathrm{TiO}_{2}$ to achieve both efficient separation of hole-electron pairs and extension of absorption spectrum beyond ultraviolet region [5].

$\mathrm{WO}_{3}$ is an fascinating semiconductor with relatively chemical stability under acidic conditions, non-toxicity, and a comparably narrow band gap $(\sim 2.4-2.8 \mathrm{eV})$ and thus able to find applications in various fields including photoeletrocatalytic processes, elertrochromic devices, dye-sensitized solar cells, and gas sensors [6-8]. Especially, it is a suitable candidate to fabricate composites with $\mathrm{TiO}_{2}$ to create heterojunction structures that facilitate the transfer and separation of photoinduced electrons and holes and hereby inhibit the recombination of charge carriers [9-11]. Accordingly, band-gap shrinkage was observed in composites on account of the formation of heterojunction structures [12].
Therefore, photocatalytic performances of $\mathrm{WO}_{3}-\mathrm{TiO}_{2}$ composites are greatly enhanced upon visible-light irradiation. Besides, the incorporation of $\mathrm{WO}_{3}$ into composites is able to increase the surface acidity since $\mathrm{WO}_{3}$ is much more acidic than $\mathrm{TiO}_{2}$ [9,13-15], and the increase of surface acidity induces the high affinity of composites to those species with unpaired electrons, such as dissolved $\mathrm{O}_{2}$, $\mathrm{H}_{2} \mathrm{O}, \mathrm{OH}^{-}$and organic contaminants, benefitting the generation of $\cdot \mathrm{O}_{2}{ }^{-}$and $\bullet \mathrm{OH}$ radicals and further decomposition of contaminants. In addition, redox reactions of $\mathrm{W}$ species (transformation between $\mathrm{W}^{6+}$ and $\mathrm{W}^{5+}$ ) in composites were deemed as another factor causing the efficient separation of hole-electron pairs [13]. As a result, $\mathrm{WO}_{3}-$ $\mathrm{TiO}_{2}$ composites have been extensively investigated for different purpose, particularly for photocatalytic processes [16-20].

It is well demonstrated that the photocatalytic capability is closely relevant to morphology and particle size of nanostructured composites that control the amount of exposed active sites and diffusion distance between charge carriers and active sites [21-24]. Loading or incorporation of composites onto a stable matrix can achieve both goals of particle sizes control and increasing adsorption of reactant molecules around active sites as a consequence of large specific surface areas of selected matrixes, likely mesoporous silicate SBA15 with ordered hexagonal morphologies, large specific surface areas, tunable pore sizes, good hydrothermal stability, and unique electron delocalization capacity by framework [25-29]. To the best of our knowledge, there is no published study concerning synthesis and photocatalytic evaluation of $\mathrm{W}$ and $\mathrm{Ti}$ species co-contained mesoporous SBA15. Herein, we reported for the first time the preparation of WTS series through a facile onestep hydrothermal approach with a purpose of obtaining enhanced catalytic performance toward degradation of dye RhB and 2,4-DCP under visible-light irradiation in this investigation.
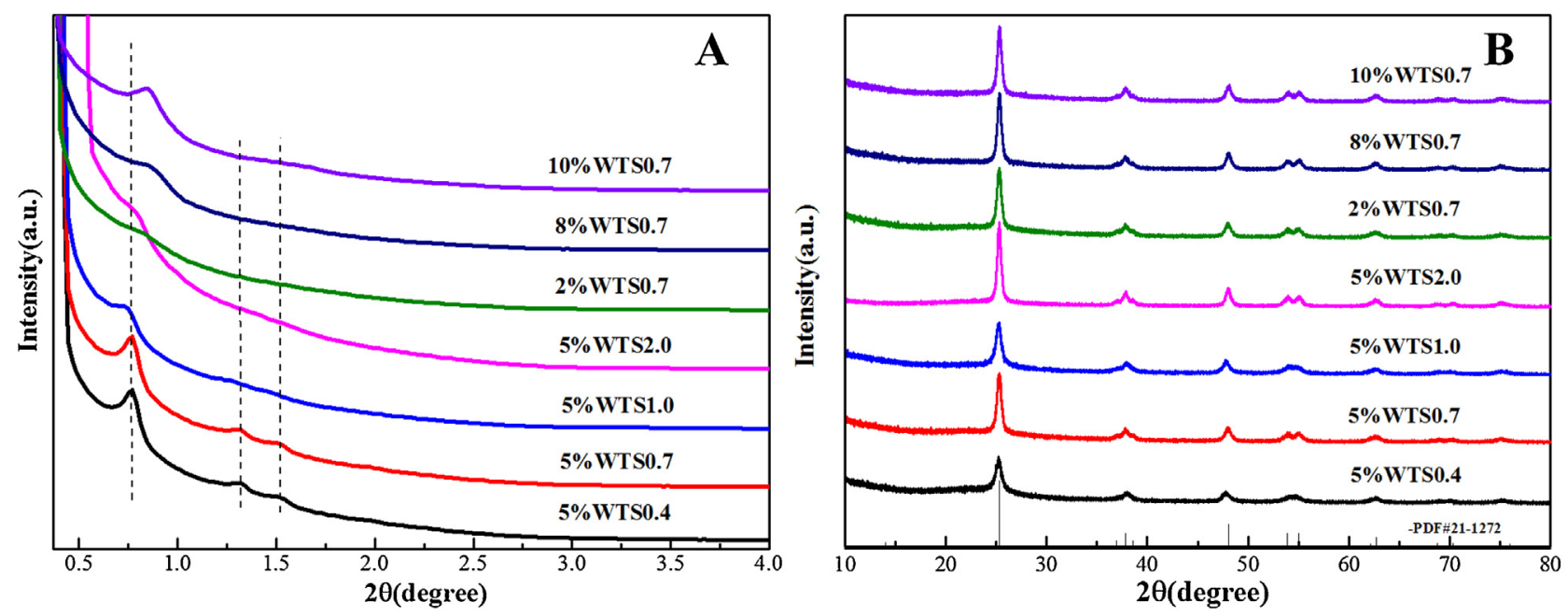

Fig. 1. Low-angle (A) and wide-angle (B) XRD patterns of WTS series.

Table 1

$S_{B E T}$ Pore volume, average pore diameter, and $\mathrm{WO}_{3} / \mathrm{TiO}_{2} / \mathrm{SiO}_{2}$ mass ratios of WTS series.

\begin{tabular}{|c|c|c|c|c|c|c|c|}
\hline \multirow[t]{2}{*}{ Samples } & \multirow[t]{2}{*}{$\mathrm{S}_{\mathrm{BET}}\left(\mathrm{m}^{2} / \mathrm{g}\right)$} & \multirow[t]{2}{*}{ Pore volume $\left(\mathrm{cm}^{3} / \mathrm{g}\right)$} & \multirow[t]{2}{*}{ Average pore diameter (nm) } & \multicolumn{2}{|c|}{ Mass ratios of $\mathrm{WO}_{3} / \mathrm{TiO}_{2}$} & Mass ratios & of $\mathrm{TiO}_{2} / \mathrm{SiO}_{2}$ \\
\hline & & & & Expected values & XPS values & Expected values & XPS values \\
\hline 2\%WTS0.7 & 494.4 & 0.958 & 7.41 & 0.02 & - & 0.7 & 0.224 \\
\hline 5\%WTS0.7 & 541.9 & 1.222 & 8.24 & 0.05 & 0.048 & 0.7 & 0.212 \\
\hline 8\%WTS0.7 & 541.0 & 1.097 & 7.43 & 0.08 & 0.077 & 0.7 & 0.198 \\
\hline 10\%WTS0.7 & 351.3 & 0.877 & 7.48 & 0.10 & 0.09 & 0.7 & 0.212 \\
\hline 5\%WTS0.4 & 314.3 & 0.838 & 8.20 & 0.05 & 0.047 & 0.4 & 0.154 \\
\hline 5\%WTS1.0 & 237.5 & 0.711 & 9.05 & 0.05 & 0.046 & 1.0 & 0.374 \\
\hline 5\%WTS2.0 & 233.4 & 0.673 & 9.01 & 0.05 & 0.056 & 2.0 & 0.420 \\
\hline $8 \%$ WS & 770.8 & 1.176 & 6.88 & & & & \\
\hline TS0.7 & 247.3 & 0.71 & 7.40 & & & & \\
\hline
\end{tabular}




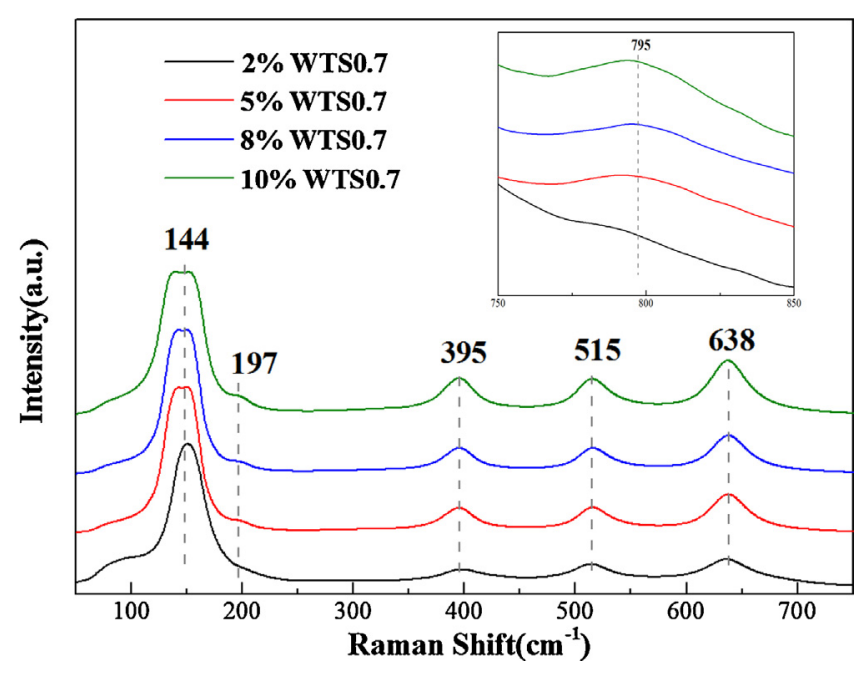

Fig. 2. Raman spectra of WTS composites.

\section{Material and methods}

\subsection{Materials}

Pluronic P123 $\left(\mathrm{PEO}_{20} \mathrm{PPO}_{70} \mathrm{PEO}_{20}\right)$ was obtained from Aldrich Chemical Co. Sodium tungstate $\left(\mathrm{Na}_{2} \mathrm{WO}_{4} \cdot 2 \mathrm{H}_{2} \mathrm{O}\right)$ was purchased from ACROS Organics. Hydrochloric acid ( $\mathrm{HCl}, 36-38 \%$, AR), titanium sulfate $\left(\mathrm{Ti}\left(\mathrm{SO}_{4}\right)_{2} \cdot 9 \mathrm{H}_{2} \mathrm{O}, \mathrm{CP}\right)$, tetraethyl orthosilicate (TEOS, $\left.\mathrm{Si}\left(\mathrm{OC}_{2} \mathrm{H}_{5}\right)_{4}, \mathrm{AR}\right)$, and other chemicals involved were purchased from Sinopharm Chemical Reagent Co., Ltd. (Shanghai, China) and used directly for experiments without any further purification. Deionized water was used throughout the experimental.

\subsection{Synthesis of $W$-Ti-SBA15 composites}

In a typical synthetic procedure, Pluronic $123(2 \mathrm{~g})$ was dissolved into an $\mathrm{HCl}$ aqueous solution $(2 \mathrm{M}, 60 \mathrm{~mL})$ to create a mixture that was added by TEOS ( $3.7 \mathrm{~g})$, and desired amount of titanic sulfate and sodium tungstate in sequence. The resultant suspension was stirred at $40^{\circ} \mathrm{C}$ for $1 \mathrm{~h}$ before transferring into a Teflon-lined stainless steel autoclave and staying at $110^{\circ} \mathrm{C}$ for $72 \mathrm{~h}$. After cooling to room temperature, a collected precipitate was washed by deionized water and absolute ethanol for several times, dried at $60^{\circ} \mathrm{C}$ overnight, and annealed at $600^{\circ} \mathrm{C}$ for $5 \mathrm{~h}$ to supply a white powder W-Ti-SBA-15. For convenience, samples with different content of $\mathrm{W}, \mathrm{Ti}, \mathrm{Si}$ were marked as $\mathrm{xWTSy}$, in which $\mathrm{x}$ and $\mathrm{y}$ referred to theoretical mass ratios of formed $\mathrm{WO}_{3} / \mathrm{TiO}_{2}$ and $\mathrm{TiO}_{2} / \mathrm{SiO}_{2}$. Samples $8 \% \mathrm{WS}$, TS0.7, and $\mathrm{WO}_{3} / \mathrm{TiO}_{2}$ were fabricated according to a same procedure above except absence of titanium, tungsten, and silicon sources, respectively. In addition, N-doped $\mathrm{TiO}_{2}$ synthesized according to a previous report [30] and a mechanically mixed 8\%WTS0.7 prepared by finely grinding a mixture of $\mathrm{WO}_{3}$ and TS0.7 with a desired mass ratio were denoted as $\mathrm{N}-\mathrm{TiO}_{2}$ and mechanically mixed WTS and then subjected to photocatalytic measurement for comparison.

\subsection{Characterization}

The morphological studies of obtained samples were measured with transmission electron microscopy (TEM, JEOL JEM-2011). X-ray diffraction (XRD) patterns were recorded on a Bruker D8 Advance X-ray diffractometer using $\mathrm{Cu} K \alpha$ radiation source ( $\lambda=1.5406 \AA$ ). The specific surface areas were measured by nitrogen adsorption-desorption isotherms at $77 \mathrm{~K}$ using a Micromeritics 3Flex apparatus. Before measurement, the samples were degassed at $383 \mathrm{~K}$ under vacuum for more than $12 \mathrm{~h}$. UV-vis diffuse reflectance spectra (UV-vis DRS) were obtained on a Shimadzu UV-2600 spectrophotometer using $\mathrm{BaSO}_{4}$ as a reference. X-ray photoelectron spectroscopy (XPS) was measured on an ESCALAB 250XI Multifunctional imaging electron spectrometer (Thermo). Binding energies were calibrated by using the containment carbon $(\mathrm{C} 1 \mathrm{~s}=284.6 \mathrm{~V})$. Raman spectra were recorded on a Bruker Senterra instrument with $532 \mathrm{~nm}$ as the exciting source from an argon ion laser.

\subsection{Photocatalytic performance}

Photocatalytic performance of as-prepared WTS composites was measured by degrading dye RhB and 2,4-DCP under visiblelight irradiation from a $300 \mathrm{~W}$ xenon lamp (CEL-HXF300, AuLight, Beijing) equipping with a $420-780 \mathrm{~nm}$ cut-off filter. The distance between the light source and the surface of reaction suspension was fixed as $20 \mathrm{~cm}$. Catalyst ( $20 \mathrm{mg}$ ) was added to an aqueous solution of $\mathrm{RhB}\left(10 \mathrm{mg} \mathrm{L}^{-1}, 80 \mathrm{~mL}\right)$ or 2,4-DCP $\left(5 \mathrm{mg} \mathrm{L}^{-1}, 80 \mathrm{~mL}\right)$ and stirred in dark for $1 \mathrm{~h}$ to reach an adsorption saturation of organic compounds onto surface of photocatalyst particles prior to irradiation. At every $30 \mathrm{~min}$ intervals, $4 \mathrm{~mL}$ aliquot was sampled, diluted, and centrifuged to remove catalyst particles. The residue concentration of RhB and 2,4-DCP was evaluated by a UV-vis spectrophotometer (Purkinje General T6) at the maximum absorption wavelength $554 \mathrm{~nm}$ and a UV-vis spectrophotometer (Shimadzu UV-2600) at the maximum absorption wavelength $281 \mathrm{~nm}$, respectively.

Effects of several active radical species on photocatalytic outcome were studied to understand photocatalytic mechanism. Specifically, $1.0 \mathrm{mM}$ isopropanol alcohol (IPA) or disodium ethylenediaminetetraacetate (EDTA-2Na) was added to detect hydroxyl radicals $\left(\bullet^{\circ} \mathrm{OH}\right)$ or produced holes $\left(\mathrm{h}^{+}\right)$[31]. $25 \mu \mathrm{M}$ nitroblue tetrazolium (NBT) was added in place of $\mathrm{RhB}$ to analyze superoxide radicals $\left(\bullet^{\circ} \mathrm{O}_{2}^{-}\right)$that might possibly generate during photocatalysis [32]. The ESR technique was further used to detect the presence of radicals $\bullet \mathrm{OH}$ and ${ }^{\circ} \mathrm{O}_{2}{ }^{-}$in system. Radicals $\bullet \mathrm{OH}$ and ${ }^{\circ} \mathrm{O}_{2}-$ could be trapped by 5,5-dimethyl-1-pyrroline- $\mathrm{N}$-oxide (DMPO) on a FA200 spectrometer when the catalyst was respectively dispersed in deionized water for DMPO- ${ }^{\bullet} \mathrm{OH}$ and methanol for DMPO- ${ }^{-} \mathrm{O}_{2}{ }^{-}$, and the settings for spectrometer were as follows: center field, $323.357 \mathrm{mT}$; microwave frequency, $9.05 \mathrm{GHz}$; power, $0.998 \mathrm{~mW}$.

The reusability of sample $8 \%$ WTS0.7 for catalytic degradation of $\mathrm{RhB}$ was performed for seven successive runs. After each run, the catalyst was centrifuged, ultrasonicated for several times, washed with deionized water and ethanol, dried overnight at $100^{\circ} \mathrm{C}$, and then was used directly for next cycle.

\section{Results and discussion}

Low-angle XRD patterns of as-synthesized WTS composites with different mass ratios of $\mathrm{WO}_{3} / \mathrm{TiO}_{2} / \mathrm{SiO}_{2}$ are depicted in Fig. $1 \mathrm{~A}$. It is distinct that low-angle $\mathrm{X}$-ray diagrams of all samples are wellresolved and exhibit three characteristic peaks corresponding to Bragg reflections on (100), (110), and (200) planes of mesoporous silicate materials, revealing the maintenance of original structure of $p 6 \mathrm{~mm}$ hexagonal symmetry [33]. In addition, the intensity of diffraction peak (100) of samples somewhat declines along with the increment of titanium additive dosage, attributing to the gradual destruction of long-range ordering of hexagonal pore structure [34] or the variation in electron density of titanium-incorporated materials, similar to tungsten incorporated MCM-41 [35]. A series of prominent diffraction peaks at nearly $25.6^{\circ}, 38.2^{\circ}, 48.4^{\circ}, 54.4^{\circ}$, $55.4^{\circ}, 63.1^{\circ}$ are found in Fig. $1 \mathrm{~B}$, corresponding to crystal planes (101), (004), (200), (105), (211), (213) of tetragonal phase of 

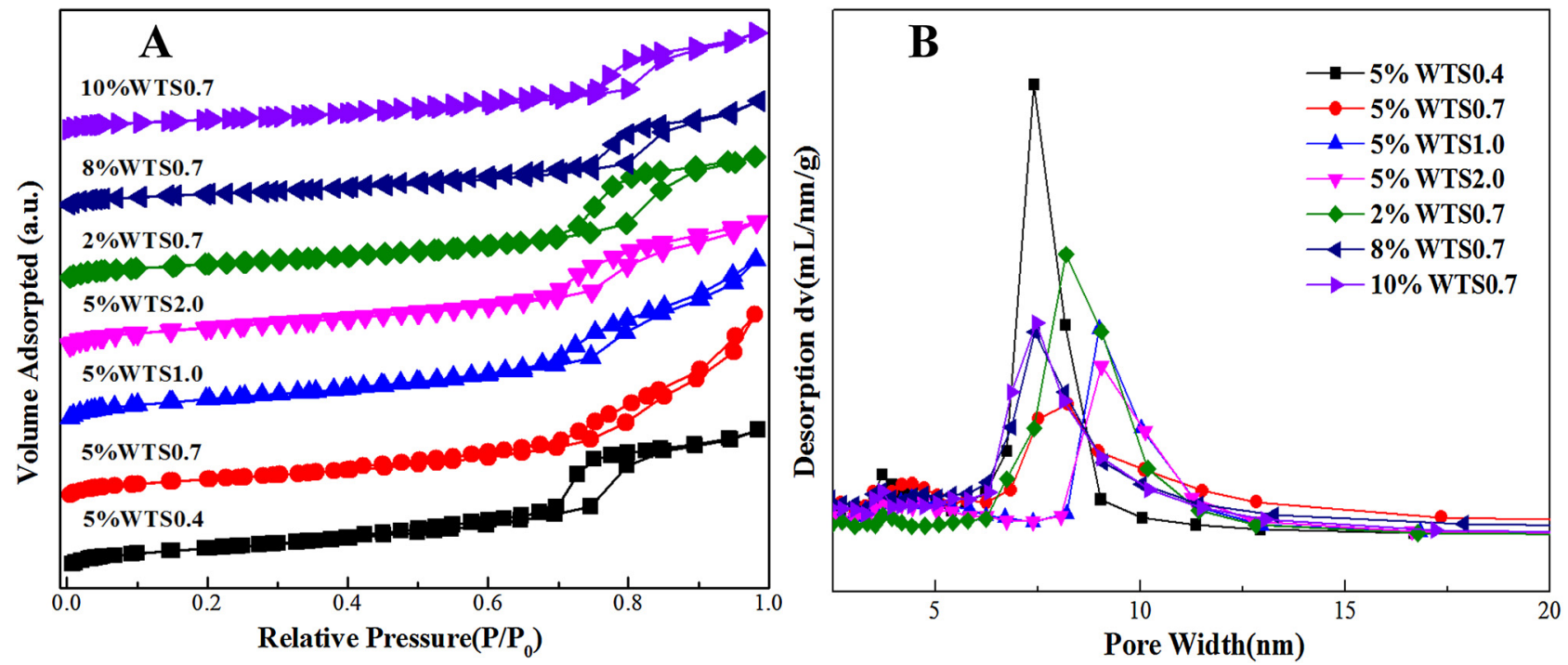

Fig. 3. N2 adsorption-desorption isotherms (A) and pore size distribution (B) of various WTS samples.

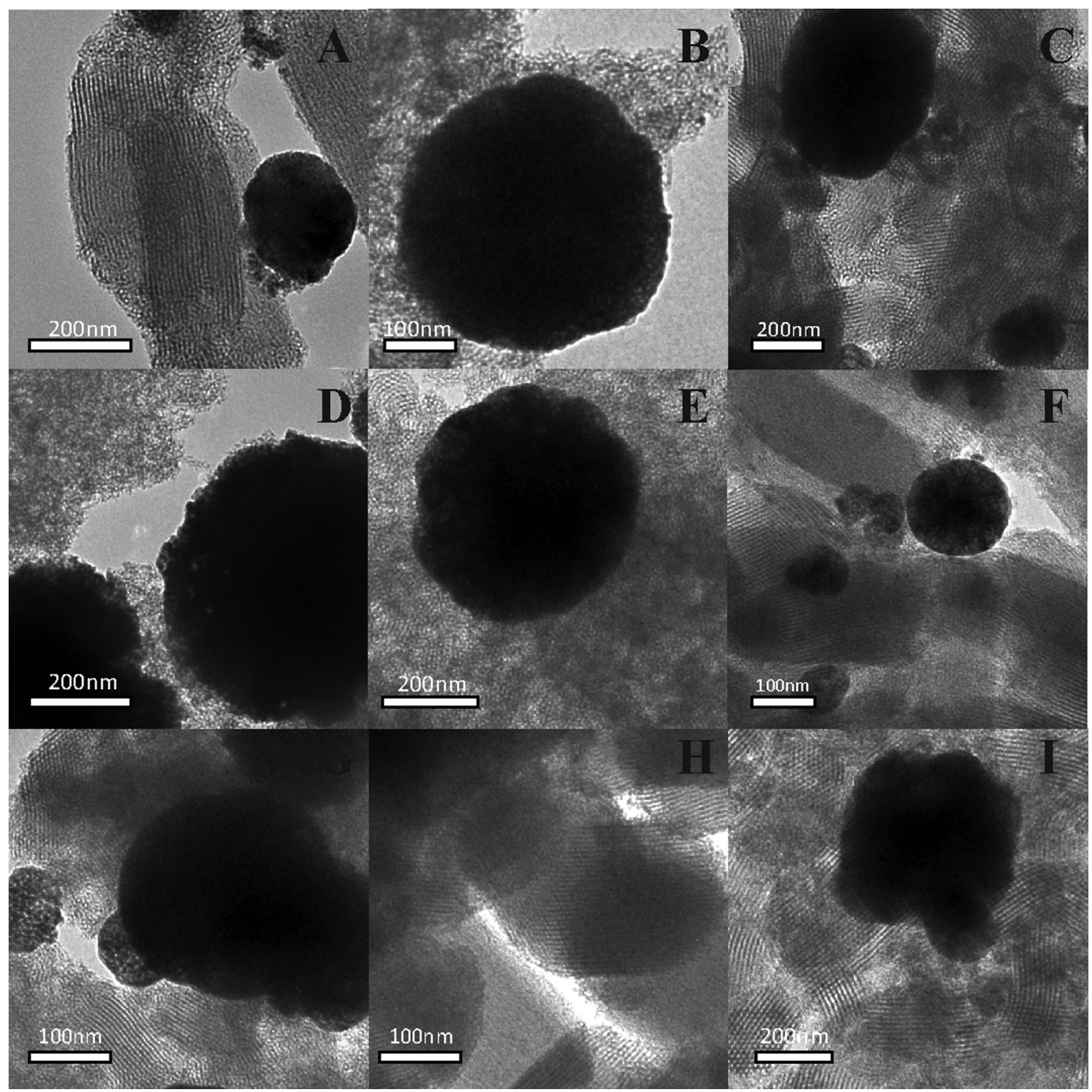

Fig. 4. TEM images of samples: (A) 5\%WTS0.4, (B) 5\%WTS0.7, (C) 5\%WTS1.0, (D) 5\%WTS2.0, (E) 2\%WTS0.7, (F) 8\%WTS0.7, (G) 10\%WTS0.7,(H) 8\%WS, and (I) TS0.7. 

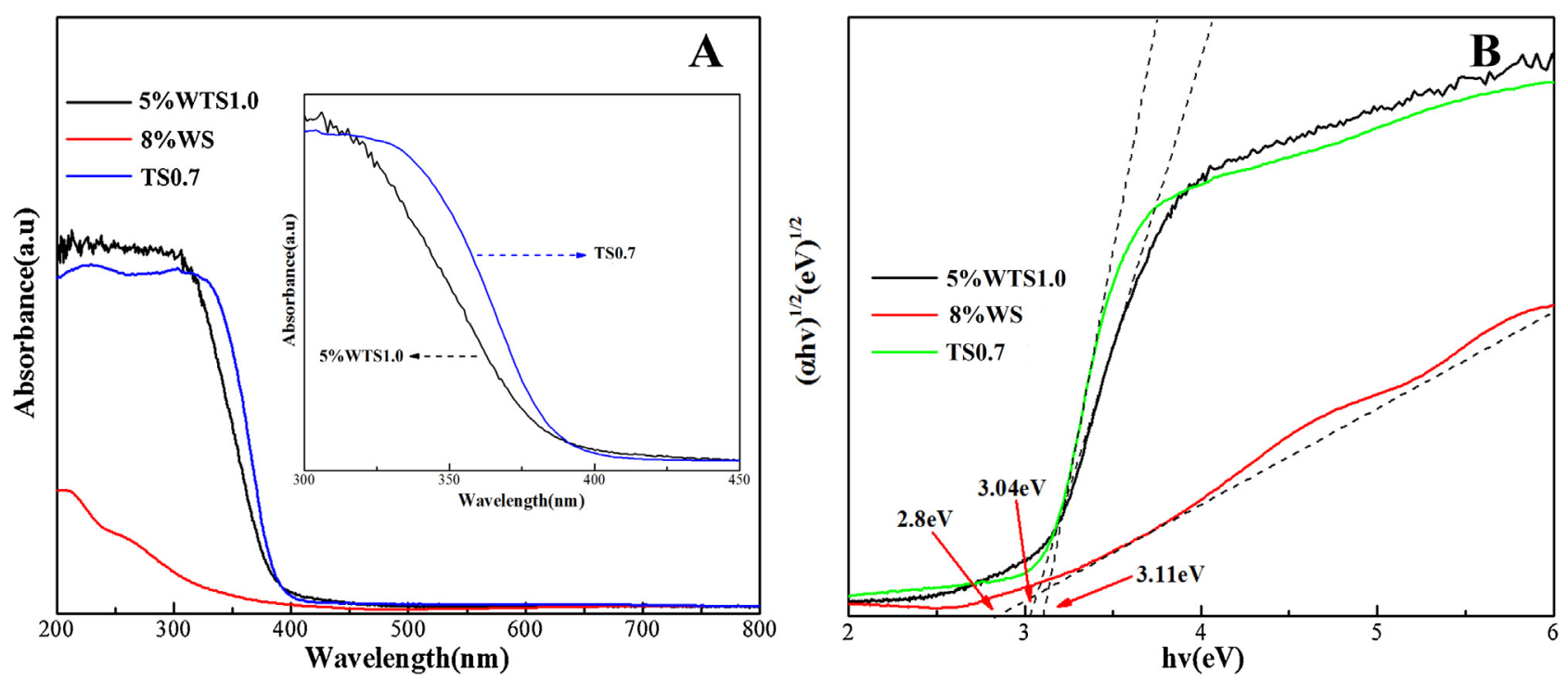

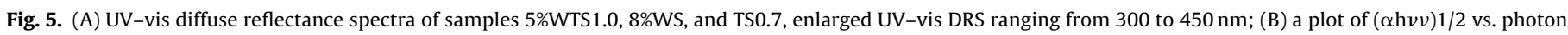
energy $(h v v)$ of corresponding samples.
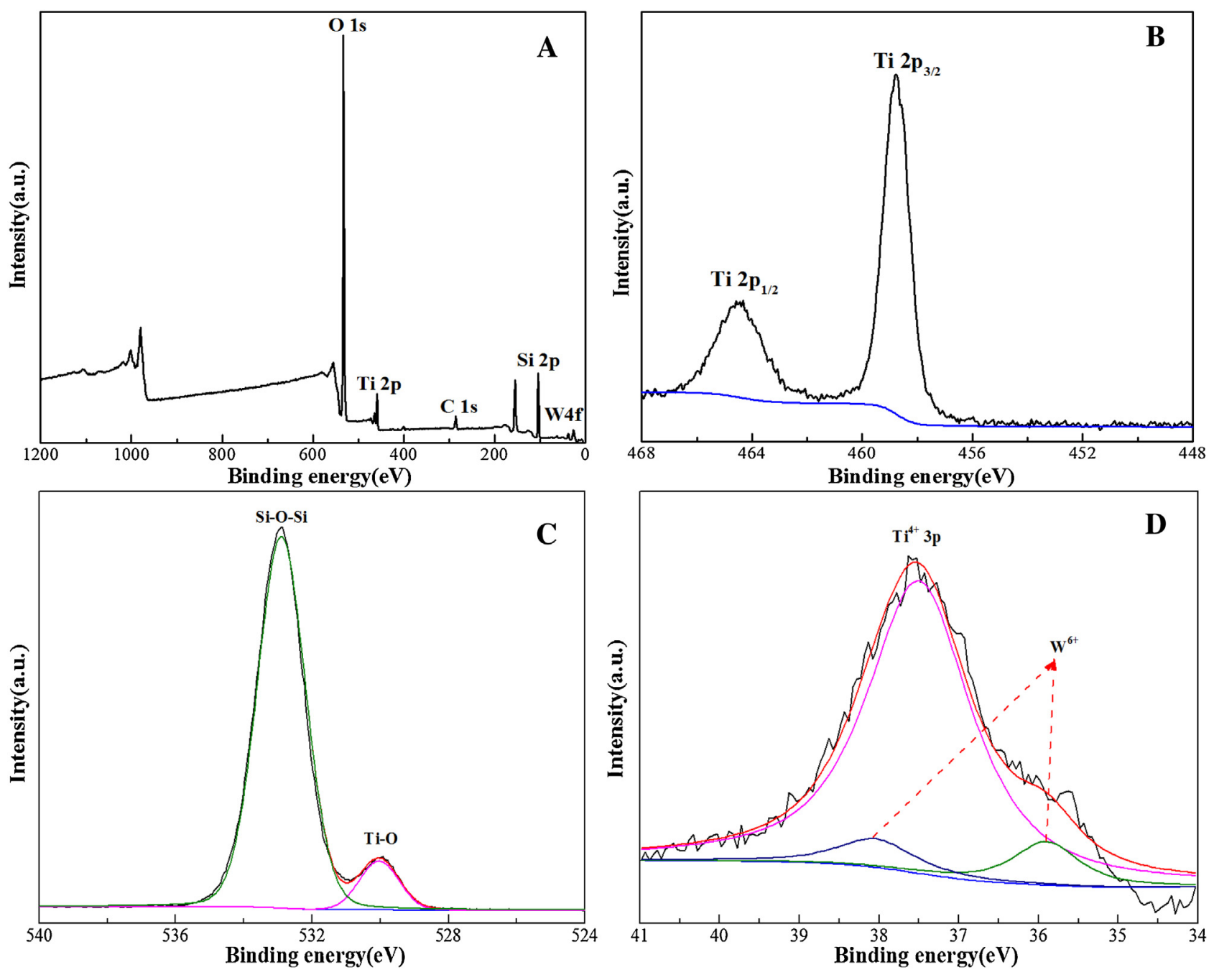

Fig. 6. XPS analysis of 8\%WTS0.7: (A) survey spectra, (B) Ti 2p, (C) O 1s, (D) W 4f.

extra-framework anatase $\mathrm{TiO}_{2}$ nanoparticles (JCPDS No. 21-1272) $[15,20]$. No diffraction peaks attributing to $\mathrm{WO}_{3}$ are detectable, possibly owing to the uniform dispersion of $\mathrm{WO}_{3}$ into $\mathrm{TiO}_{2}$ matrix or substitution of $\mathrm{Si}^{4+}$ by tungsten ions as a consequence of similar 

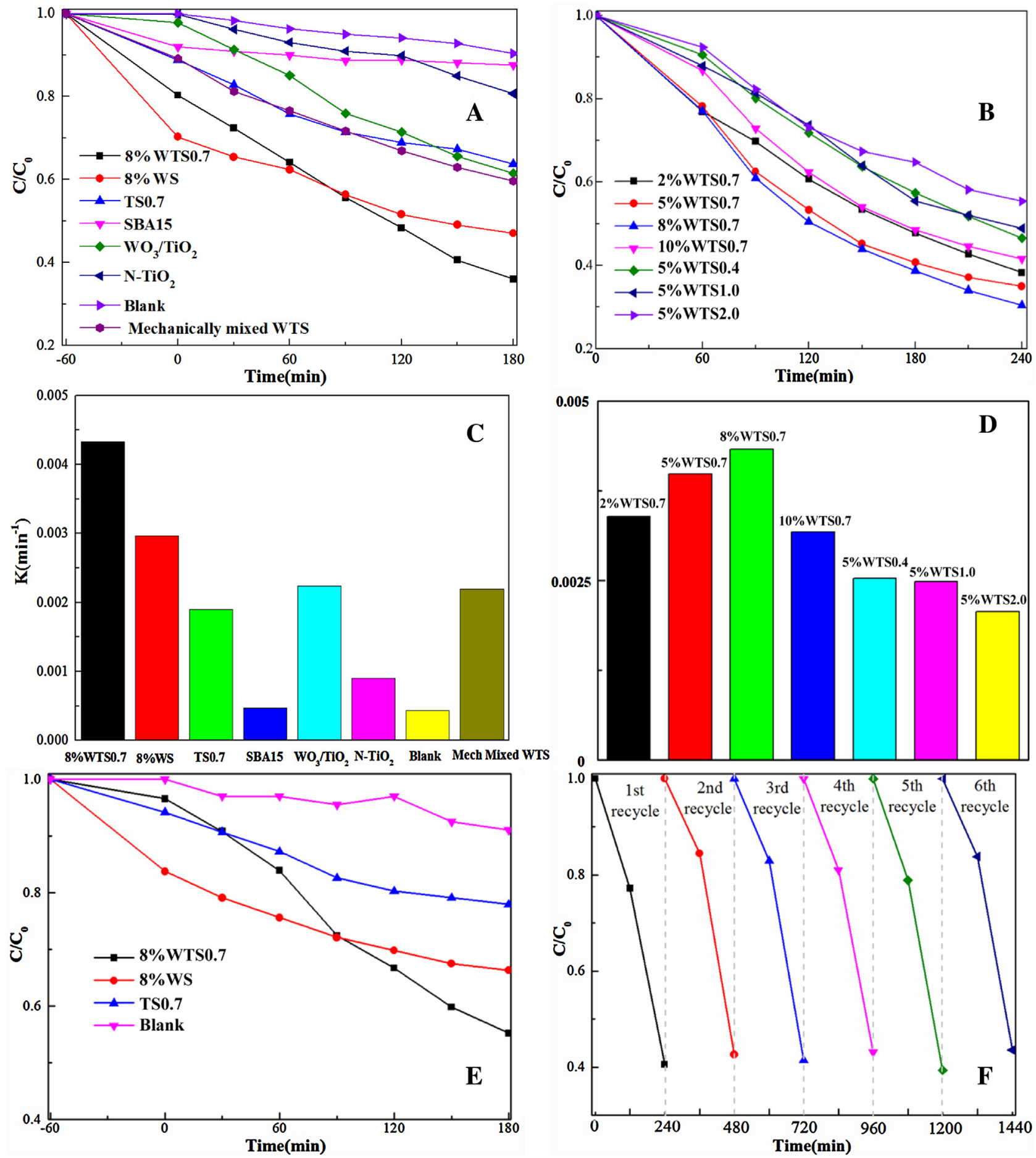

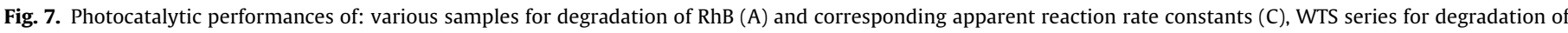

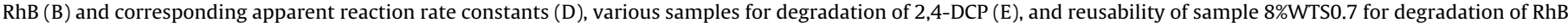
degradation $(\mathrm{F})$.

ion radius of both [16]. In addition, there are no peaks at nearly $20.45^{\circ}$ and $22.83^{\circ}$ in spectra, revealing the absence of solid solution of $\mathrm{W}_{\mathrm{x}} \mathrm{Ti}_{1-\mathrm{x}} \mathrm{O}_{2}$, as reported [36].

The comparison of $\mathrm{WO}_{3} / \mathrm{TiO}_{2}$ and $\mathrm{TiO}_{2} / \mathrm{SiO}_{2}$ mass ratios in composites between expected and XPS analytical values is shown in Table $1 . \mathrm{WO}_{3} / \mathrm{TiO}_{2}$ mass ratios by XPS analysis are in good agreement with those calculated from initial titanium and tungsten contents in gel. However, $\mathrm{TiO}_{2} / \mathrm{SiO}_{2}$ mass ratios in samples by XPS analysis are much lower than those calculated values. This result can be explained as follows. The titanium and tungsten species added are well mixed to make a mixture in solution. A large fraction of titanium and tungsten species mixture tends to stay in solution because of the large solubility of titanium species and low content of tungsten species in acidic medium [37] and only a small fraction of species mixture with the initial mass ratios integrates with silicate to produce WTS composites. In addition, it is generally realized that the optimal Ti/Si molar ratio in initial gel should be set below 0.015 so as to produce high-quality Ti-SBA-15 [38]. The 

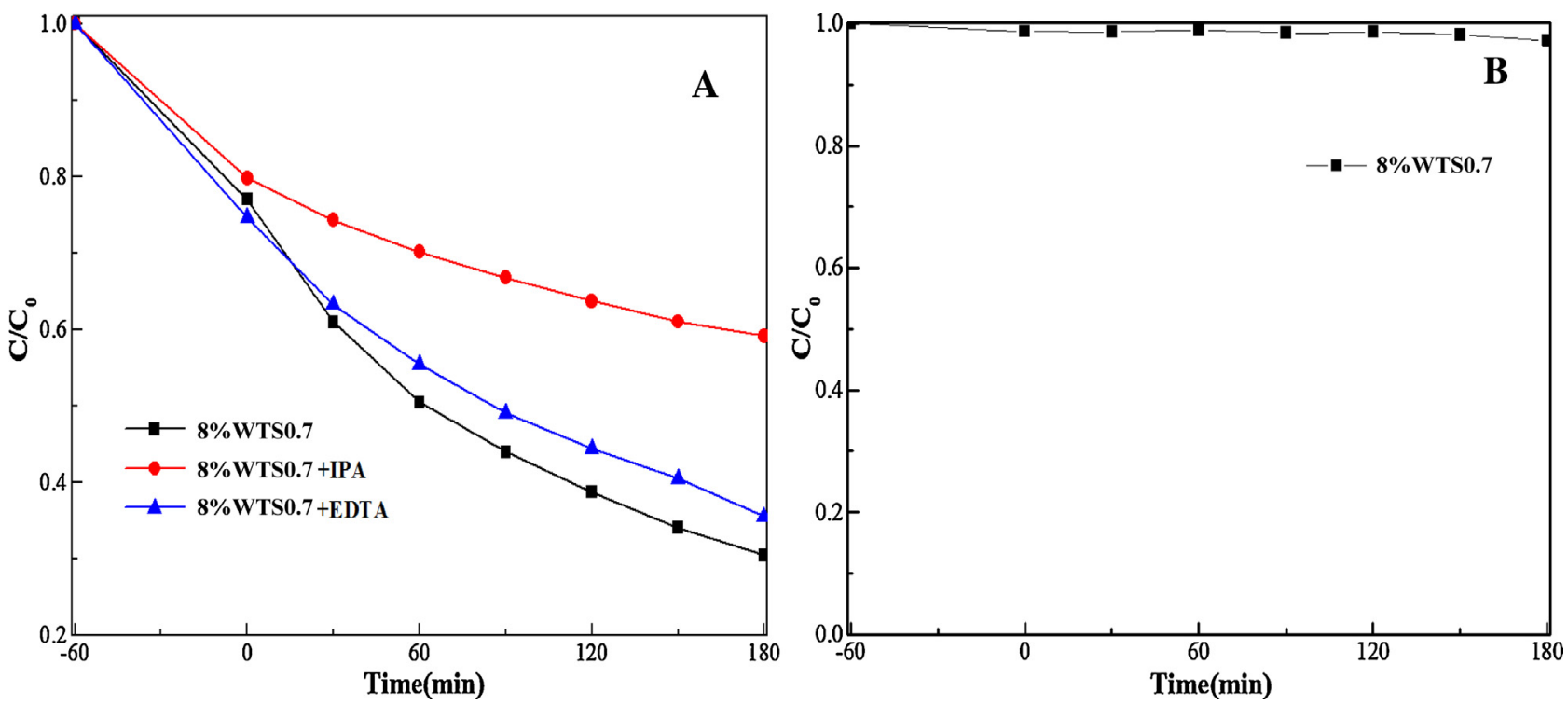

Fig. 8. Active species trapping experiments (A), and transformation percentage of NBT concentration (B) using sample 8\%WTS0.7.
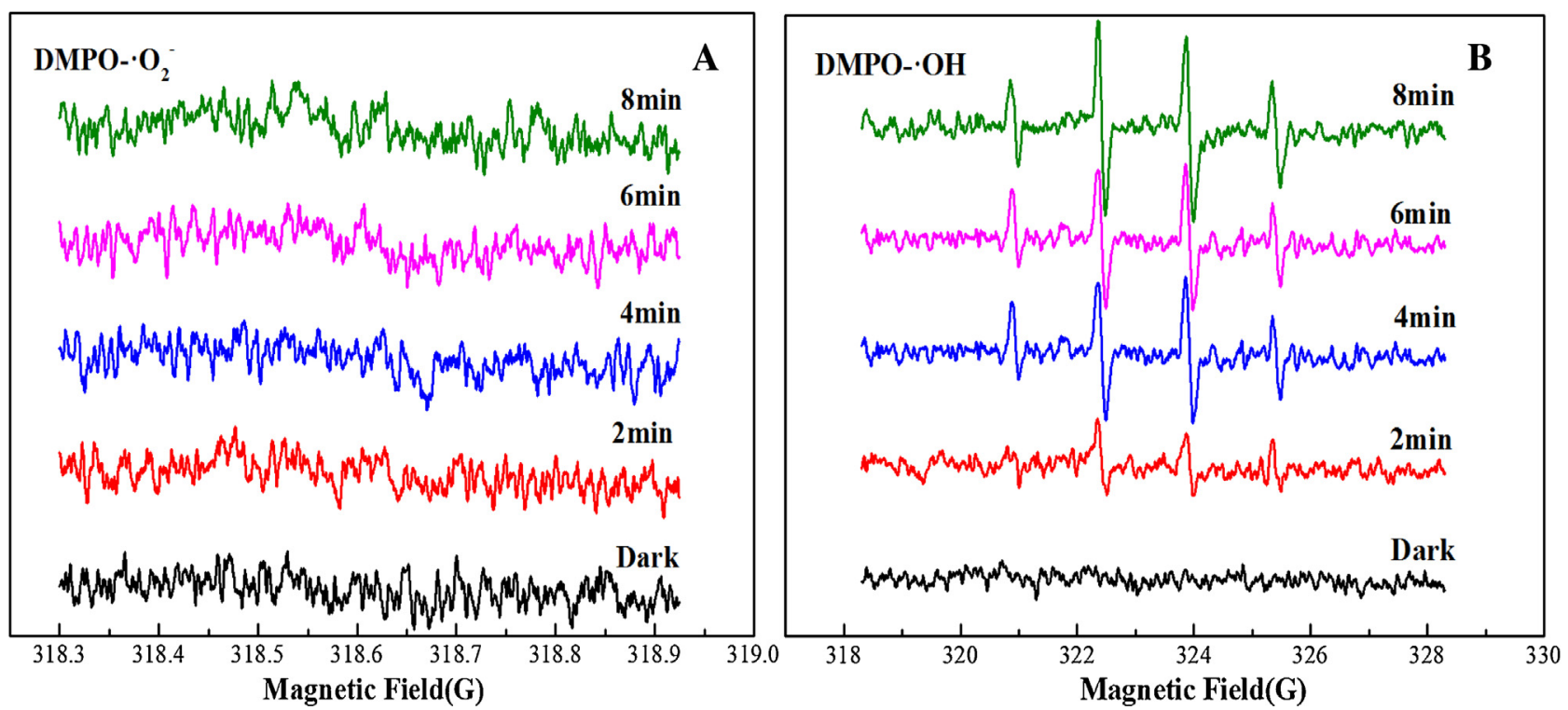

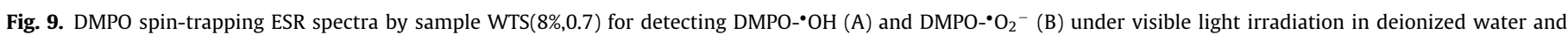
methanol, respectively.

titanium component tends to transform from incorporated species in silicon skeleton to extra-framework anatase nanoparticles when excess dosage of titanium additive is involved [39], which has been evidenced by wide-angle XRD patterns and can be further identified through Raman spectra in Fig. 2. Strong signals at 144, 197, 395,515 , and $638 \mathrm{~cm}^{-1}$ are present in all composites and unambiguously relevant to anatase phase $\mathrm{TiO}_{2}$ in nanocrystal form [40]. Since these samples are of same mass ratio of $\mathrm{TiO}_{2} / \mathrm{SiO}_{2}$, the intensity of those corresponding peaks is almost identical. A weak band centered at around $795 \mathrm{~cm}^{-1}$ in inset of Fig. 2 with the intensity gradually enhanced with the increase of $\mathrm{WO}_{3} / \mathrm{TiO}_{2}$ mass ratios is assigned to the $\mathrm{O}-\mathrm{W}-\mathrm{O}$ stretching vibrations [41], revealing the presence of trace mount of monoclinic $\mathrm{WO}_{3}$ in composites.

Fig. 3A represents $\mathrm{N}_{2}$ adsorption-desorption isotherms of obtained WTS series to further characterize their structural variation. Obviously, all composites exhibit the type IV isotherm except 5\%WTS0.7 basing upon the IUPAC classification and type H1 hysteresis loop at relative pressure $\mathrm{P} / \mathrm{P}_{0}$ ranging from 0.5 to 0.8 , indicative of uniform mesoporous structures with hexagonal cylindrical pores at the capillary condensation step [42]. In addition, the hysteresis loop widely covers at $\mathrm{P} / \mathrm{P}_{0}$ region beyond $\mathrm{P} / \mathrm{P}_{0}=0.8$, associating to the intra-aggregate voids of $\mathrm{TiO}_{2}$ nanocrystallines [43]. Specific surface area, pore volume and pore diameter of all WTS samples are listed in Table 1. As observed, specific surface area values of samples with constant tungsten species amount obviously increase first and then dramatically reduce when the $\mathrm{TiO}_{2} / \mathrm{SiO}_{2}$ mass ratio surpasses 0.7. Similarly, the variation of $\mathrm{WO}_{3} / \mathrm{TiO}_{2}$ mass ratio up to $8 \%$ has little effect on specific surface area of samples and then significantly decline. In addition, the average pore diameter of samples is keeps almost constant at fixed $\mathrm{TiO}_{2} / \mathrm{SiO}_{2}$ mass ratios and gradually enlarged with the increment of titanium spices contents in Fig. 3B. Therefore, both titanium species incorporated into silica matrix and aggregated as anatase microcrystals outside channels lead to the variation of $S_{B E T}$ and average pore diameter of obtained composites. 
High resolution TEM images depicted in Fig. 4, supplying visualization of textural properties of as-prepared WTS samples with different contents of tungsten and titanium species, reveal that WTS series are composed of well-ordered hexagonal arrays of mesoporous matrix and regularly-shaped spherical aggregates with size between 0.1 and $0.6 \mu \mathrm{m}$ that was mainly controlled by the amount of titanium species involved. These black spherical aggregates that decorated on surface of SBA15 matrix turn out to be anatase $\mathrm{TiO}_{2}$ microcrystals, confirmed by wide-angle XRD patterns, Raman spectra, and following XPS analyses. The coexistence of mesoporous structure with large specific surface areas and catalyst particles is beneficial to absorb contaminant molecules around active sites and further decompose them efficiently. Accordingly, the photocatalytic behavior of samples is significantly enhanced. In addition, a similar TEM image of TS0.7 to those of WTS series and a distinct TEM image without black lump substance for $8 \% \mathrm{WS}$ are shown in Fig. 4I and $\mathrm{H}$, respectively.

UV-vis diffuse reflectance spectra of samples 5\%WTS1.0, 8\%WS, and TS0.7 were measured and shown in Fig. 5A. Sample 8\%WS exhibits a weak absorption in UV and visible-light region, thanks to the low content of tungsten species contained. In addition, the spectrum of sample TS0.7 is dominated by the edge relative to the $\mathrm{O}^{-2}-\mathrm{Ti}^{+4}$ charge transition ranging from 300 to $380 \mathrm{~nm}$ of anatase $\mathrm{TiO}_{2}$ [20]. The adsorption edge of sample 5\%WTS0.7 is slightly redshifted in comparison to that of sample TS0.7. However, sample 5\%WTS0.7 shows an additional broad and weak absorption (inset of Fig. 5A) in ranging from 400 to $450 \mathrm{~nm}$ that is ascribed to an $\mathrm{O}^{-2}-\mathrm{W}^{+6}$ charge transfer transition [14]. Corresponding band gap energies $\left(E_{g}\right)$ of above samples were calculated by an empirical equation $\alpha h v=A(h v-E g)^{n / 2}$ as $2.8 \mathrm{eV}, 3.11 \mathrm{eV}$, and $3.04 \mathrm{eV}$ for $8 \% \mathrm{WS}$, TS0.7, and 5\%WTS0.7, respectively, as seen in Fig. 5B. The shrinkage of band gap energies facilitates the excitation of semiconductors to produce electron-hole pairs, thus benefiting to the improvement of photocatalytic degradation efficiency. UV-vis DRS spectra of other samples were depicted in Fig. A.1 .

The chemical composition and surface valence states of sample $8 \%$ WTS0.7 were measured by XPS analysis and are displayed in Fig. 6. Peak positions in all spectra were calibrated using $\mathrm{C} 1 \mathrm{~s}$ at $284.6 \mathrm{eV}$. The XPS survey spectrum in Fig. 6A reveals the coexistence of elements Ti, W, O, and C, which is in agreement with our expectation. Two intense peaks at $458.5 \mathrm{eV}$ and $464.2 \mathrm{eV}$ in Fig. $6 \mathrm{~B}$ are relevant to the $\mathrm{Ti} 2 \mathrm{p}_{3 / 2}-\mathrm{Ti} 2 \mathrm{p}_{1 / 2}$ spin-orbit components of $\mathrm{Ti}^{4+}$ species in $\mathrm{TiO}_{2}$ lattice $[11,19]$. The lack of binding-energy peaks corresponding to $\mathrm{Ti}^{3+}$ species indicates the absence of surface defects [19]. The O 1s spectrum in Fig. 6C can be fitted well with doublet peaks cantered at $529.8 \mathrm{eV}$ and $532.8 \mathrm{eV}$. The former is indexed to $\mathrm{O}-\mathrm{Ti}$ bonds in $\mathrm{TiO}_{2}$ network [44] and the later positioned at $532.8 \mathrm{eV}$ is assigned to $\mathrm{Si}-\mathrm{O}-\mathrm{Si}$ bonds in SBA-15 matrix [45]. No peaks originating from $\mathrm{O}-\mathrm{W}$ bonds are undetectable, which is mainly due to the low content of tungsten oxide species. The XPS spectrum is deconvoluted to three peaks at binding energies of about $37.3 \mathrm{eV}, 35.8 \mathrm{eV}$, and $37.9 \mathrm{eV}$, as observed in Fig. 6D. The first located at $37.3 \mathrm{eV}$ is ascribed to $\mathrm{Ti}^{4+}$ ( $\mathrm{Ti} 3 \mathrm{p}$ ) species in anatase $\mathrm{TiO}_{2}$ phase $[11,46]$. The last two due to the spin-orbit splitting of W (4f) components demonstrate the formation of $\mathrm{WO}_{3}$ in composite [47]. The binding energy difference $5.7 \mathrm{eV}$ and $2.1 \mathrm{eV}$ of Ti $2 \mathrm{p}$ and W4f are in good accordance with the theory of level splitting and those values reported for $\mathrm{TiO}_{2}$ and $\mathrm{WO}_{3}$ substances [18].

Photocatalytic activities of as-prepared WTS series were evaluated by decomposition of dye RhB and 2,4-DCP under visible-light irradiation. It is obvious that in Fig. 7A the direct pyrolysis of RhB without catalysts is almost neglectable and $\mathrm{N}-\mathrm{TiO}_{2}$ shows a quite low photocatalytic behavior. Bare SBA15 exhibits slight adsorption ability over RhB. Samples $\mathrm{WO}_{3} / \mathrm{TiO}_{2}$ and TSO.7 can destruct similar amount of RhB (40\%) after $3 \mathrm{~h}$ irradiation, except that sample TS0.7 had a relatively high adsorption ability resulted by the presence of SBA15 matrix. Interestingly, sample 8\%WS shows the highest adsorption capability among all samples containing SBA matrix because of the much more acidic nature of $\mathrm{WO}_{3}$ than that of $\mathrm{TiO}_{2}$ [9]. However, with the progress of photocatalysis, sample 8\%WTS0.7 with both components of $\mathrm{WO}_{3}$ and $\mathrm{TiO}_{2}$ anchored on surface of SBA15 support tends to show a satisfactory photocatalytic performance that is even higher than $8 \% \mathrm{WS}$ after $1.5 \mathrm{~h}$. As a result, it is tempting to conclude that the composite is able to achieve excellent photocatalytic outcome under identical conditions, especially with the aid of an appropriate support like SBA15. It should be noted that photocatalytic ability of mechanically ground sample is much lower than that of sample $8 \%$ WTS0.7 and quite close to that of sample TS0.7 in Fig. 7A, implying the possible existence of heterojunction structures in composite 8\%WTS0.7, instead of a simple physical mixture. Photocatalytic performance of WTS series with different contents of tungsten and titanium species was subsequently measured and is represented in Fig. 7B. With the increase of titanium species content at the fixed $\mathrm{WO}_{3} / \mathrm{TiO}_{2}$ mass ratio $5 \%$, the removal efficiency of catalysts rises first and then falls and best photocatalyst candidate turns to be 5\%WTS0.7. A similar trend is observed with the variation of tungsten species at the fixed $\mathrm{TiO}_{2} / \mathrm{SiO}_{2}$ mass ratio 0.7 . Eventually, the sample $8 \%$ WTS0.7 is deemed as the most efficient candidate among WTS series. The apparent reaction rate constants were calculated by means of a pseudo-first-order kinetic equation simplified from the well-known Langmuir-Hinshelwood kinetic model in quite diluted solutions [12], as shown in Fig. 7C and D. As expected, among WTS series sample 8\%WTS0.7 is of the largest apparent reaction rate constant that is approximately $1.2,2.1,2.2$, and 5.7 times as high as $8 \% \mathrm{WS}, \mathrm{TS} 0.7, \mathrm{WO}_{3} / \mathrm{TiO}_{2}$, and $\mathrm{N}-\mathrm{TiO}_{2}$, respectively. The enhancement of photocatalytic performance of WTS series is mainly attributed to the synergistic effect existed not only between both components of $\mathrm{TiO}_{2}$ and $\mathrm{WO}_{3}$, but also between semiconductor composites and silicate matrix. Specifically, the incorporation of $\mathrm{WO}_{3}$ improves the adsorption ability of $\mathrm{TiO}_{2}$ towards dissolved $\mathrm{O}_{2}, \mathrm{H}_{2} \mathrm{O}, \mathrm{OH}^{-}$and contaminant molecules, owning to the much stronger acidity nature and thus more affinity of $\mathrm{WO}_{3}$ than that of $\mathrm{TiO}_{2}$ [15]. In addition, energy levels of both semiconductors are well-matched and easily overlap in band structures, benefiting to create heterojunction structures to efficiently separate electrons and holes and thus enhance photocatalytic performance [17]. Furthermore, as an appropriate mesoporous support, SBA15 promotes the photocatalytic degradation efficiency by exerting both roles: the enhancement of contaminant molecules adsorption by interacting with abundant hydroxyl groups on surface with large specific surface area [48] and the efficient separation of electron-hole pairs through the electron delocalization capacity through SBA-15 framework [29]. Finally, a suitable mass ratio of tungsten species/titanium species is necessary to obtain optimal photocatalytic degradation efficiency. Below that, the insufficient amount of tungsten species is unable to form heterostructures and beyond that, excess amount of tungsten species tends to aggregate to bulk $\mathrm{WO}_{3}$ phase that shows quite low photocatalytic performance as a result of rapid recombination of charge carriers [6]. As a result, with a considerable specific surface area, favorable optical property, and suitable mass ratio of two species, the sample 8\%WTS0.7 bears the best catalytic efficiency towards degradation of dye RhB among all tested candidates.

It is generally accepted that RhB molecules are sensitive to visible light and a dye-sensitized photodegradation path, instead of photocatalytic degradation, may possibly occur [49]. To exclude this possibility, 2,4-DCP, a colorless organic pollutant, was typically chosen to confirm the visible-light-driven photocatalytic destruction ability of sample 8\%WTS0.7, as shown in Fig. 7E. Apparently, the photocatalytic degradation efficiency of 2,4-DCP reaches to $45 \%$ after $3 \mathrm{~h}$ that is nearly twice as large as that from sample TS0.7 by a 


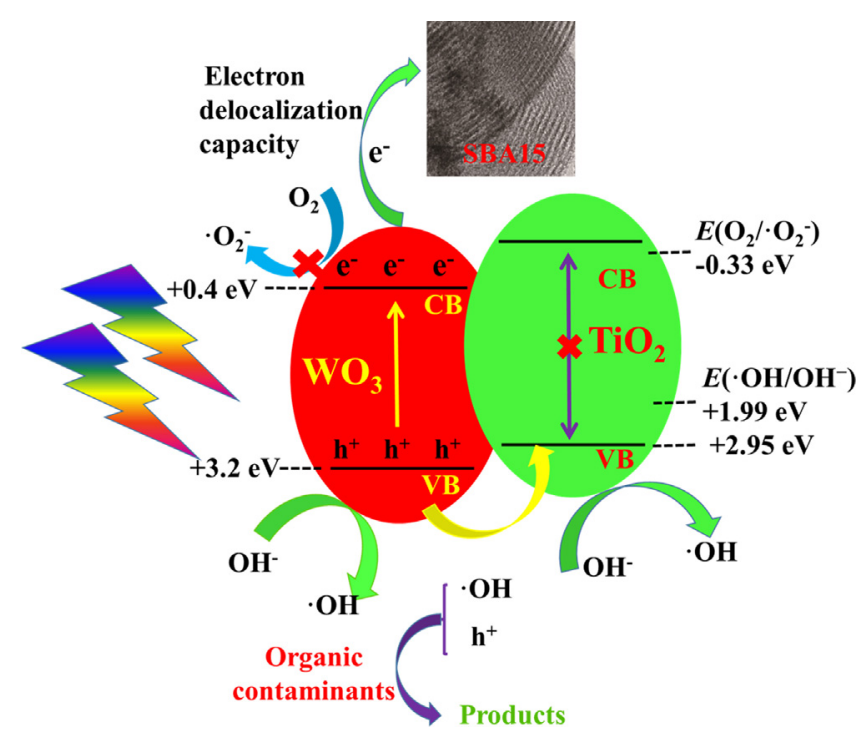

Scheme 1. Proposed mechanism of WTS composites upon visible light irradiation.

dye-sensitized photodegradation route. The corresponding apparent reaction rate constants were shown in Fig. A.2 .

The reusability and durability of WTS series are also crucial factors for their industrial applications, which were further evaluated by successive photocatalytic entries of sample 8\%WTS0.7 in Fig. 7F. Satisfactory photocatalytic ability could be maintained without obvious catalytic deactivation, even though it had been reused for six times. It should be noted that the sample 8\%WTS0.7 was easily recycled via a simple centrifugation, rinse with water and ethanol, and dryness under an ambient temperature. As a result, WTS series are effective and stable visible-light-driven catalysts and can be potentially applied in environmental protection and remediation in future.

As usual, catalytic degradation of organic pollutants upon irradiation relays on several active radical species, such as $h^{+}, \bullet \mathrm{H}$, and $\bullet_{2}{ }^{-}$. Therefore, active radicals trapping experiments were accomplished over 8\%WTS0.7 to identify radical species that might generate and exert oxidation roles in photocatalytic process. As depicted in Fig. 8A, a slight decrease is found in photocatalytic result upon addition of EDTA-2Na, indicating that holes have a minor effect on photocatalytic reactions. However, the addition of IPA intensively hindered the degradation capability, implying that radicals ${ }^{\bullet} \mathrm{OH}$ are dominant active radicals. The amount of ${ }^{\bullet} \mathrm{O}_{2}{ }^{-}$formed during photocatalytic reactions can be quantitatively calculated through the concentration change of NBT in aqueous solutions [32]. In our experimental, the invariableness of NBT concentration is observable in Fig. 7B, implying the impossible generation of radicals $\cdot \mathrm{O}_{2}{ }^{-}$. In order to further detect ${ }^{\bullet} \mathrm{OH}$ and ${ }^{\bullet} \mathrm{O}_{2}{ }^{-}$radicals, ESR spectra of DMPO- ${ }^{-} \mathrm{O}_{2}{ }^{-}$and DMPO- ${ }^{\bullet} \mathrm{OH}$ adducts on sample $8 \%$ WTS0.7 in methanol and deionized water were measured and shown in Fig. 9. Four characteristic peaks of DMPO- ${ }^{\circ} \mathrm{OH}$ adducts appears and no signals belonging to $\mathrm{DMPO}-{ }^{\bullet} \mathrm{O}_{2}{ }^{-}$adducts are found under visible-light irradiation, implying that radicals $\bullet \mathrm{OH}$ are predominate oxidative species rather than holes or ${ }^{\bullet} \mathrm{O}_{2}{ }^{-}$during photocatalysis.

Basing upon above analysis, a possible photocatalytic mechanism is eventually proposed in Scheme 1 . Under visible-light irradiation $(420 \mathrm{~nm}<\lambda<780 \mathrm{~nm}), W_{3}$ is easily excited to produce photogenerated electron-hole pairs due to its suitable bandgap energy and $\mathrm{TiO}_{2}$ keeps in ground state. Excited electrons in $\mathrm{WO}_{3}$ move to conduction band with a band edge potential $\left(E_{C B}\right)$ of $+0.4 \mathrm{eV}$ and holes remain on valence band with a band edge potential $\left(E_{V B}\right)$ of $+3.2 \mathrm{eV}$ [50]. $E_{V B}$ of $\mathrm{WO}_{3}$ is much more positive than the $E_{V B}(+2.95 \mathrm{eV})$ of $\mathrm{TiO}_{2}$ and hereby the holes on valence band of
$\mathrm{WO}_{3}$ tends to transfer across interfaces to that of $\mathrm{TiO}_{2}$ [50]. Electrons stay on conduction band of $\mathrm{WO}_{3}$ since the $E_{C B}$ of $\mathrm{WO}_{3}$ is less negative than that of $\mathrm{TiO}_{2}$. Accordingly, the transfer and separation of produced charge carriers is successfully achieved, which is quite beneficial for enhancement of photocatalytic degradation efficiency. In addition, electrons on conduction band of $\mathrm{WO}_{3}$ are insufficient to reduce dissolved molecular oxygen on surface to supply radicals ${ }^{\circ} \mathrm{O}_{2}^{-}$, attributing to the much more positive $E_{C B}$ $(+0.4 \mathrm{eV})$ than $E\left(\mathrm{O}_{2} /{ }^{\bullet} \mathrm{O}_{2}{ }^{-}\right)(-0.33 \mathrm{eV})[51]$. These electrons can be dispersed and efficiently removed by means of the electron delocalization capacity of silicate framework [29]. On the contrary, both $E_{V B}(+3.2 \mathrm{eV})$ of $\mathrm{WO}_{3}$ and $E_{V B}(+2.95 \mathrm{eV})$ of $\mathrm{TiO}_{2}$ are much more positive than $E\left({ }^{\bullet} \mathrm{OH} / \mathrm{OH}^{-}\right)(+1.99 \mathrm{eV})$ and radicals ${ }^{\bullet} \mathrm{OH}$ are hereby prone to generate through the oxidation of water or $\mathrm{OH}^{-}$anions adsorbed around active sites [51]. As a result, holes and radicals - $\mathrm{OH}$ participate the photocatalytic process and radicals $\bullet \mathrm{OH}$ exert a major effect on the photocatalytic process.

\section{Conclusions}

In this investigation, we reported the preparation, characterization, and photocatalytic evaluation of WTS series under visible-light irradiation. These WTS composites were subjected to the photocatalytic degradation of dye RhB and 2,4-DCP and exhibited improved catalytic behavior comparing with those counterparts containing only single component of $\mathrm{WO}_{3}$ or $\mathrm{TiO}_{2}$. Specifically, the best candidate, sample 8\%WTS0.7, showed the highest photocatalytic degradation efficiency with an apparent reaction rate constant that was nearly $1.2,2.1,2.2$, and 5.7 times as high as $8 \% \mathrm{WS}$, TS0.7, $\mathrm{WO}_{3} / \mathrm{TiO}_{2}$, and $\mathrm{N}-\mathrm{TiO}_{2}$. The enhancement of photocatalytic performance was mainly relevant to well-matched electron structures of both $\mathrm{WO}_{3}$ and $\mathrm{TiO}_{2}$ and strengthened adsorption ability originating from the modification with $\mathrm{WO}_{3}$ with high acidity and SBA15 with large specific surface areas. Radicals ${ }^{\circ} \mathrm{OH}$ were deemed as major species in photocatalytic system and a possible photocatalytic mechanism was eventually proposed.

\section{Acknowledgements}

We are grateful to the National Natural Science Foundation of China (No. 21207089), the project-sponsored by SRF for ROCS, SEM., the Hujiang Foundation of China (No. B14003), the Innovation Program of Shanghai Municipal Education Commission (No. 14ZZ136), and the Natural Science Foundation of Shanghai (No. 13ZR1427600) for financial support.

\section{Appendix B. Supplementary data}

Supplementary data associated with this article can be found, in the online version, at http://dx.doi.org/10.1016/j.colsurfa.2016.04. 013.

\section{References}

[1] C. Chen, W. Ma, J. Zhao, Semiconductor-mediated photodegradation of pollutants under visible-light irradiation, Chem. Soc. Rev. 39 (2010) 4206-4219.

[2] A. Kubacka, M. Fernandez-Garcia, G. Colon, Advanced nanoarchitectures for solar photocatalytic applications, Chem. Rev. 112 (2012) 1555-1614.

[3] H. Wang, L. Zhang, Z. Chen, J. Hu, S. Li, Z. Wang, J. Liu, X. Wang, Semiconductor heterojunction photocatalysts: design, construction, and photocatalytic performances, Chem. Soc. Rev. 43 (2014) 5234-5244.

[4] H. Dong, G. Zeng, L. Tang, C. Fan, C. Zhang, X. He, Y. He, An overview on limitations of TiO2-based particles for photocatalytic degradation of organic pollutants and the corresponding countermeasures, Water Res. 79 (2015) $128-146$.

[5] X. Chen, S.S. Mao, Titanium dioxide nanomaterials: synthesis, properties, modifications, and applications, Chem. Rev. 107 (2007) 2891-2959. 
[6] A. Hiskia, A. Mylonas, E. Papaconstantinou, Comparison of the photoredox properties of polyoxometallates and semiconducting particles, Chem. Soc. Rev. 30 (2001) 62-69.

[7] M.S. Bazarjani, M. Hojamberdiev, K. Morita, G. Zhu, G. Cherkashinin, C. Fasel, T. Herrmann, H. Breitzke, A. Gurlo, R. Riedel, Visible light photocatalysis with $c-\mathrm{WO}_{3-\mathrm{x}} / \mathrm{WO}_{(3) \mathrm{x}} \mathrm{H}_{(2)} \mathrm{O}$ nanoheterostructures in situ formed in mesoporous polycarbosilane-siloxane polymer, J. Am. Chem. Soc. 135 (2013) 4467-4475

[8] A. Yan, C. Xie, F. Huang, S. Zhang, S. Zhang, An efficient method to modulate the structure, morphology and properties of $\mathrm{WO}_{3}$ through niobium doping, J. Alloys Compd. 610 (2014) 132-137.

[9] K.K. Akurati, A. Vital, J.P. Dellemann, K. Michalow, T. Graule, D. Fetti, A. Baiker, Flame-made $\mathrm{WO}_{3} / \mathrm{TiO}_{2}$ nanoparticles: relation between surface acidity, structure and photocatalytic activity, Appl. Catal. B 79 (2008) 53-62.

[10] Y. Liu, C.S. Xie, H.Y. Li, H. Chen, T. Zou, D.W. Zeng, Improvement of gaseous pollutant photocatalysis with $\mathrm{WO}_{3} / \mathrm{TiO}_{2}$ heterojunctional-electrical layered system, J. Hazard. Mater. 196 (2011) 52-58.

[11] A.O.T. Patrocinio, L.F. Paula, R.M. Paniago, J. Freitag, D.W. Bahnemann, Layer-by-layer $\mathrm{TiO}_{2} / \mathrm{WO}_{3}$ thin films as efficient photocatalytic self-cleaning surfaces, ACS Appl. Mater. Interfaces 6 (2014) 16859-16866.

[12] N.A. Ramos-Delgado, M.A. Gracia-Pinilla, L. Maya-Trevino, L. Hinojosa-Reyes, J.L. Guzman-Mar, A. Hernandez-Ramirez, Solar photocatalytic activity of TiO modified with $\mathrm{WO}_{3}$ on the degradation of an organophosphorus pesticide, J. Hazard. Mater. 263 (2013) 36-44.

[13] Y. Yuan, Y. Zhao, H. Li, Y. Li, X. Gao, C. Zheng, J. Zhang, Electrospun metal oxide-tiO $\mathrm{H}_{2}$ nanofibers for elemental mercury removal from flue gas, J. Hazard. Mater. 227 (2012) 427-435.

[14] A.K.L. Sajjad, S. Shamaila, B.Z. Tian, F. Chen, J.L. Zhang, One step activation of $\mathrm{WO}_{\mathrm{x}} / \mathrm{TiO}_{2}$ nanocomposites with enhanced photocatalytic activity, Appl. Catal. B 91 (2009) 397-405.

[15] A.K.L. Sajjad, S. Shamaila, B. Tian, F. Chen, J. Zhang, Comparative studies of operational parameters of degradation of azo dyes in visible light by highly efficient $\mathrm{WOx} / \mathrm{TiO}_{2}$ photocatalyst, J. Hazard. Mater. 177 (2010) 781-791.

[16] O. Lorret, D. Francova, G. Waldner, N. Stelzer, W-doped titania nanoparticles for UV and visible-light photocatalytic reactions, Appl. Catal. B 91 (2009) 39-46.

[17] V. Puddu, R. Mokaya, G.L. Puma, Novel one step hydrothermal synthesis of $\mathrm{TiO}_{2} / \mathrm{WO}_{3}$ nanocomposites with enhanced photocatalytic activity, Chem. Commun. (2007) 4749-4751.

[18] D. Su, J. Wang, Y. Tang, C. Liu, L. Liu, X. Han, Constructing $\mathrm{WO}_{3} / \mathrm{TiO}_{2}$ composite structure towards sufficient use of solar energy, Chem. Commun. 47 (2011) 4231-4233.

[19] A. Alonso-Tellez, D. Robert, V. Keller, N. Keller, $\mathrm{H}_{2} \mathrm{~S}$ photocatalytic oxidation over $\mathrm{WO}_{3} / \mathrm{TiO}_{2}$ hombikat UV100, Environ. Sci. Pollut. Res. 21 (2014) 3503-3514.

[20] K. Lv, J. Li, X. Qing, W. Li, Q. Chen, Synthesis and photo-degradation application of $\mathrm{WO}_{3} / \mathrm{TiO}_{2}$ hollow spheres, J. Hazard. Mater. 189 (2011) 329-335.

[21] Y. Liu, J. Li, W. Li, Y. Yang, Y. Li, Q. Chen, Enhancement of the photoelectrochemical performance of $\mathrm{WO}_{3}$ vertical arrays film for solar water splitting by gadolinium doping, J. Phys. Chem. C 119 (2015) 14834-14842.

[22] E. Mena, A. Rey, S. Contreras, F.J. Beltran, Visible light photocatalytic ozonation of DEET in the presence of different forms of $\mathrm{WO}_{3}$, Catal. Today 252 (2015) 100-106.

[23] D. Tanaka, Y. Oaki, H. Imai, Enhanced photocatalytic activity of quantum-confined tungsten trioxide nanoparticles in mesoporous silica, Chem. Commun. 46 (2010) 5286-5288.

[24] G. Xi, B. Yue, J. Cao, J. Ye, $\mathrm{Fe}_{3} \mathrm{O}_{4} / \mathrm{WO}_{3}$ Hierarchical core-shell structure: high-performance and recyclable visible-light photocatalysis, Chem.-Eur. J. 17 (2011) 5145-5154.

[25] D.Y. Zhao, J.L. Feng, Q.S. Huo, N. Melosh, G.H. Fredrickson, B.F. Chmelka, G.D. Stucky, Triblock copolymer syntheses of mesoporous silica with periodic 50 to 300 angstrom pores, Science 279 (1998) 548-552.

[26] D.Y. Zhao, Q.S. Huo, J.L. Feng, B.F. Chmelka, G.D. Stucky, Nonionic triblock and star diblock copolymer and oligomeric surfactant syntheses of highly ordered hydrothermally stable, mesoporous silica structures, J. Am. Chem. Soc. 120 (1998) 6024-6036.

[27] S. Perathoner, P. Lanzafame, R. Passalacqua, G. Centi, R. Schlogl, D.S. Su, Use of mesoporous SBA-15 for nanostructuring titania for photocatalytic applications, Microporous Mesoporous Mater. 90 (2006) 347-361.

[28] W. Wang, M. Song, Photocatalytic activity of titania-containing mesoporous SBA-15 silica, Microporous Mesoporous Mater. 96 (2006) 255-261.

[29] M.V.P. Sharma, V.D. Kumari, M. Subrahmanyam, $\mathrm{TiO}_{2}$ Supported over SBA-15: an efficient photocatalyst for the pesticide degradation using solar light, Chemosphere 73 (2008) 1562-1569.

[30] T. Li, L. Zhao, Y. He, J. Cai, M. Luo, J. Lin, Synthesis of $g-\mathrm{C}_{3} \mathrm{~N}_{4} / \mathrm{SmVO}_{4}$ composite photocatalyst with improved visible light photocatalytic activities in RhB degradation, Appl. Catal. B 129 (2013) 255-263.
[31] X. Hu, T. Mohamood, W. Ma, C. Chen, J. Zhao, Oxidative decomposition of rhodamine $\mathrm{B}$ dye in the presence of $\mathrm{VO}^{2+}$ and/or Pt(IV) under visible light irradiation: $n$-deethylation, chromophore cleavage, and mineralization, J. Phys. Chem. B 110 (2006) 26012-26018.

[32] L. Ye, J. Liu, Z. Jiang, T. Peng, L. Zan, Facets coupling of BiOBr-g- $\mathrm{C}_{3} \mathrm{~N}_{4}$ composite photocatalyst for enhanced visible-light-driven photocatalytic activity, Appl. Catal. B 142 (2013) 1-7.

[33] J.E. Herrera, J.H. Kwak, J.Z. Hu, Y. Wang, C.H.F. Peden, J. Macht, E. Iglesia, Synthesis characterization, and catalytic function of novel highly dispersed tungsten oxide catalysts on inesoporous silica, J. Catal. 239 (2006) 200-211.

[34] D.R. Sahu, L.Y. Hong, S.-C. Wang, J.-L. Huang, Synthesis, analysis and characterization of ordered mesoporous $\mathrm{TiO}_{2} / \mathrm{SBA}-15$ matrix: effect of calcination temperature, Microporous Mesoporous Mater. 117 (2009) 640-649.

[35] T.I. Bhuiyan, P. Arudra, M.N. Akhtar, A.M. Aitani, R.H. Abudawoud, M.A. Al-Yami, S.S. Al-Khattaf, Metathesis of 2-butene to propylene over W-mesoporous molecular sieves: a comparative study between tungsten containing MCM-41 and SBA-15, Appl. Catal. A 467 (2013) 224-234.

[36] X.Z. Li, F.B. Li, C.L. Yang, W.K. Ge, Photocatalytic activity of $\mathrm{WO}_{\mathrm{x}}-\mathrm{TiO}_{2}$ under visible light irradiation, J. Photochem. Photobiol. A 141 (2001) 209-217.

[37] Y. Chen, Y. Huang, J. Xiu, X. Han, X. Bao, Direct synthesis, characterization and catalytic activity of titanium-substituted SBA-15 mesoporous molecular sieves, Appl. Catal. A 273 (2004) 185-191.

[38] M. Wan, X. Sun, S. Liu, J. Ma, J. Zhu, Versatile drug releaser derived from the Ti-substituted mesoporous silica SBA-15, Microporous Mesoporous Mater. 199 (2014) 40-49.

[39] K. Witte, A.M. Busuioc, V. Meynen, M. Mertens, N. Bilba, G. Tendeloo, P. Cool, E.F. Vansant, Influence of the synthesis parameters of $\mathrm{TiO}_{2}-\mathrm{SBA}-15$ materials on the adsorption and photodegradation of rhodamine-6G, Microporous Mesoporous Mater. 110 (2008) 100-110.

[40] L. Yang, Z. Si, D. Weng, Y. Yao, Synthesis: characterization and photocatalytic activity of porous $\mathrm{WO}_{3} / \mathrm{TiO}_{2}$ hollow microspheres, Appl. Surf. Sci. 313 (2014) 470-478.

[41] N.A. Ramos-Delgado, M.A. Gracia-Pinilla, L. Maya-Treviňo, L. Hinojosa-Reyesa, J.L. Guzman-Mara, A. Hernández-Ramírez, Solar photocatalytic activity of $\mathrm{TiO}_{2}$ modified with $\mathrm{WO}_{3}$ on the degradation of an organophosphorus pesticide, J. Hazard. Mater. 263P (2013) 36-44.

[42] J.C. Hu, Y.D. Wang, L.F. Chen, R. Richards, W.M. Yang, Z.C. Liu, W. Xu, Synthesis and characterization of tungsten-substituted SBA-15: an enhanced catalyst for 1-butene metathesis, Microporous Mesoporous Mater. 93 (2006) 158-163.

[43] S. Chen, T. Mochizuki, Y. Abe, M. Toba, Y. Yoshimura, Ti-incorporated SBA-15 mesoporous silica as an efficient and robust Lewis solid acid catalyst for the production of high-quality biodiesel fuels, Appl. Catal. A 148-149 (2014) 344-356.

[44] M. Grandcolas, T. Cottineau, A. Louvet, N. Keller, V. Keller, Solar light-activated photocatalytic degradation of gas phase diethylsulfide on $\mathrm{WO}_{3}$-modified $\mathrm{TiO}_{2}$ nanotubes, Appl. Catal. B 138 (2013) 128-140.

[45] K. Ketpang, M. Kim, S. Kim, S. Shanmugam, High performance catalyst for electrochemical hydrogen evolution reaction based on $\mathrm{SiO}_{2} / \mathrm{WO}_{3-\mathrm{x}}$ nanofacets, Int. J. Hydrogen Energy 38 (2013) 9732-9740.

[46] A. Rey, P. Garcia-Munoz, M.D. Hernandez-Alonso, E. Mena, S. Garcia-Rodriguez, F.J. Beltran, $\mathrm{WO}_{3}-\mathrm{TiO}_{2}$ based catalysts for the simulated solar radiation assisted photocatalytic ozonation of emerging contaminants in a municipal wastewater treatment plant effluent, Appl. Catal. B 154 (2014) 274-284.

[47] Y. Cao, J. Wang, M. Kang, Y. Zhu, Efficient synthesis of ethylene glycol from cellulose over Ni- $\mathrm{WO}_{3} / \mathrm{SBA}-15$ catalysts, J. Mol. Catal. A 381 (2014) 46-53.

[48] F. Chang, G. Wang, Y. Xie, M. Zhang, J. Zhang, H.-J. Yang, X. Hu, Synthesis of $\mathrm{TiO}_{2}$ nanoparticles on mesoporous aluminosilicate Al-sBA-15 in supercritical $\mathrm{CO}_{2}$ for photocatalytic decolorization of methylene blue, Ceram. Int. 39 (2013) 3823-3829.

[49] J.J. He, G. Benko, F. Korodi, T. Polivka, R. Lomoth, B. Akermark, L.C. Sun, A. Hagfeldt, V. Sundstrom, Modified phthalocyanines for efficient near-iR sensitization of nanostructured $\mathrm{TiO}_{2}$ electrode, J. Am. Chem. Soc. 124 (2002) 4922-4932.

[50] D. Tsukamoto, M. Ikeda, Y. Shiraishi, T. Hara, N. Ichikuni, S. Tanaka, T. Hirai, Selective photocatalytic oxidation of alcohols to aldehydes in water by $\mathrm{TiO}_{2}$ partially coated with $\mathrm{WO}_{3}$, Chem. - Eur. J. 17 (2011) 9816-9824.

[51] N. Boonprakob, N. Wetchakun, S. Phanichphant, D. Waxler, P. Sherrell, A. Nattestad, J. Chen, B. Inceesungvorn, Enhanced visible-light photocatalytic activity of $g-\mathrm{C}_{3} \mathrm{~N}_{4} / \mathrm{TiO}_{2}$ films, J. Colloid Interface Sci. 417 (2014) 402-409. 